\title{
A novel prognostic prediction model based on seven immune-related RNAs for predicting overall survival of patients in early cervical squamous cell carcinoma
}

\author{
Rui Qin ${ }^{\dagger}$, Lu CaO $^{\dagger}$, Cong Ye, Junrong Wang ${ }^{*}$ (I) and Ziqian Sun ${ }^{*}$
}

\begin{abstract}
Background: In this study, we aimed to mine immune-related RNAs expressed in early cervical squamous cell carcinoma to construct prognostic prediction models.

Methods: The RNA sequencing data of 309 cervical squamous cell carcinoma (CSCC) cases, including data of individuals with available clinical information, were obtained from The Cancer Genome Atlas (TCGA) database. We included 181 early-stage CSCC tumor samples with clinical survival and prognosis information (training dataset). Then, we downloaded the GSE44001 gene expression profile data from the National Center for Biotechnology Information Gene Expression Omnibus (validation dataset). Gene ontology annotation and the Kyoto Encyclopedia of Genes and Genomes pathway analyses were used to analyze the biological functions of differentially expressed immunerelated genes (DEIRGs). We established protein-protein interactions and competing endogenous RNA networks using Cytoscape. Using the Kaplan-Meier method, we evaluated the association between the high- and low-risk groups and the actual survival and prognosis information. Our univariate and multivariate Cox regression analyses screened for independent prognostic factors.

Results: We identified seven prognosis-related signature genes (RBAKDN, CXCL2, ZAP70, CLEC2D, CD27, KLRB1, VCAM1), the expression of which was markedly associated with overall survival (OS) in CSCC patients. Also, the risk score of the seven-gene signature discripted superior ability to categorize CSCC patients into high-risk and low-risk groups, with a observablydifferent OS in the training and validation datasets. We screened two independent prognostic factors (Pathologic $\mathrm{N}$ and prognostic score model status) that correlated significantly by univariate and multivariate Cox regression analyses in the TCGA dataset. To further explore the potential mechanism of immune-related genes, we observed associated essential high-risk genes with a cytokine-cytokine receptor interaction.
\end{abstract}

Conclusions: This study established an immune-related RNA signature, which provided a reliable prognostic tool and may be of great significance for determining immune-related biomarkers in CSCC.

Keywords: Early cervical squamous cell carcinoma, Immune-related RNAs signature, Prognostic prediction model

*Correspondence: jrwang@mail.ju.edu.cn; sunziqian@mail.ju.edu.cn ${ }^{\dagger}$ Rui Qin and Lu Cao are co-first authors

Department of Obstetrics and Gynecology, The Third Hospital of Jilin University, No 126, Xiantai Street, Changchun, Jilin 130033, People's Republic of China

\section{Background}

Cervical cancer $(\mathrm{CC})$ is the second primary cause of death for women worldwide, accounting for more than 260,000 deaths each year (1). Cervical squamous cell carcinoma (CSCC) is the most common type of CC (2). Cervical intraepithelial neoplasia (CIN) is a 
precancerous lesion that is strongly related to $\mathrm{CC}$ and includes CIN I-III, each of which reflects the successive progression of CC (3). The early clinical symptoms of CC are often undetectable. Therefore, it is crucial to find markers of early-stage $\mathrm{CC}$ to improve the prevention and treatment of this disease. Biomarker discovery is a key to the early diagnosis of $\mathrm{CC}$ and improvements in cure and survival rates.

Recently, immunotherapy has been proved to be a vigorous modality to treat multifarious conditions, given that our understanding of immune system function has improved in essence (4). As a Human Papillomavirus (HPV)-driven cancer, $\mathrm{CC}$ appears to be at least partly mediated by the immune system. Checkpoint immunotherapy has shown significant efficacy in lung, bladder, renal, and head and neck cancers (5). CC will hopefully, at some point, be considered a tumor that benefits from immunotherapeutic agents. Zhao et al. (6) found that MMP1 may be a novel biomarker for immunotherapy and prognostic assessment of patients with CC. Karpathiou et al. (7) indicated that PD-L1 and CTLA-4 immune cell expression was associated with lymph node metastasis and are, therefore, potential therapeutic targets in CC. Wang et al. (8) found that immune system-related genes referred to the $\mathrm{T}$ cell receptor (TCR) signaling pathway are associated with the overall survival (OS) of CSCC patients. Previous studies reported that immune checkpoints are initiated by ligand-receptor interactions that are simply blocked by antibodies or modulated by recombinant forms of ligands or receptors. Thus, these immune checkpoints are attractive drug targets for cancer therapeutics (9). Furthermore, Yu et al. (10) analyzed the gene expression data from The Cancer Genome Atlas (TCGA) and constructed a risk model based on 26 DElncRNAs. The results indicated that the risk prediction model had a properly high accuracy to predict the prognosis of CSCC patients, suggesting that these DElncRNAs were possibly related to CSCC prognosis. Zhou et al. (11) established a regression model by CSCC gene expression, the prediction accuracy of which for CSCC was high. Although previous studies have identified a number of gene markers in the occurrence and recurrence of CSCC, further research is needed on the impact of gene characteristics on OS survival and prognosis.

In our study, we first analyzed the transcriptome to determine the expression level characteristics of earlystage CSCC cases in TCGA database. After assessing immune-related genes, we screened the RNAs closely related to CSCC and the immune system. The results allowed us to construct a prognostic model based on prognosis-related RNAs.

\section{Methods}

Data collection and preprocessing

As of March 12, 2020, the RNA sequencing data of 309 patients with CSCC, including individuals whose clinical information was available, were downloaded from TCGA. After analyzing the corresponding clinical data, only the early-stage CSCC tumor samples, marked as "stage I" and "stage II" in the "Pathologic stage" category, were retained. Finally, we included 181 early-stage CSCC tumor samples with clinical survival-related prognostic information. These cases served as the training dataset to construct a prognostic model. Therefore, we downloaded the GSE44001 gene expression profile data from the National Center for Biotechnology Information Gene Expression Omnibus (http://www.ncbi.nlm.nih.gov/geo/) (17) based on the platform of the GPL14951 (18) Illumina HumanHT-12 WG-DASL V4.0 R2 Expression BeadChip. The GSE44001 dataset included 300 CC samples with survival-related prognostic information; these cases served as the validation dataset to construct the prognostic model.

\section{Screening of differentially expressed RNAs and differentially expressed immune-regulated genes}

We used $4528 \operatorname{lncRNAs}$ and 19,194 protein-coding genes in the HUGO Gene Nomenclature Committee (http:// www.genenames.org/) (20) to identify the lncRNAs and mRNAs in the expression profile. Then, we divided the cancer cases into the poor prognostic group (A group: cases with a survival time less than three years and death) or the good prognostic group (B group: cases with a survival time greater than five years) (21). Next, limma package (Version 3.34.7, https://bioconductor.org/packages/ release/bioc/html/limma.html) (22) was used to screen the differentially expressed RNAs (DERs) in the poor prognostic and good prognostic groups using the false discovery rate (FDR) threshold of $<0.05$ and $|\log 2 \mathrm{FC}|>1$ (2 times). According to the expression value of the DERs in the training dataset, the heatmap package in $\mathrm{R}$ 3.4.1 (Version 1.0.8; https://cran.r-project.org/web/packages/ pheatmap/index.html) (23) was used to perform bidirectional hierarchical clustering on the expression of DER values based on the centered Pearson correlation algorithm (24).

Moreover, in the AmiGO 2 (http://amigo.geneontolo gy.org/amigo) database, "immune" was used as the keyword to search the biological processes related to immunity, after which we downloaded the genes involved in immune-related biological processes. We also downloaded all the related pathways and genes involved in the "immune" entry from the KEGG database. We obtained differentially expressed immune-regulated genes 
(DEIRGs) by crossing the previously acquired DEGs list with the list of immune-related genes.

\section{Construction of the co-expression network}

We used the cor.test function in R3.4.1 (https://stat.ethz. $\mathrm{ch} / \mathrm{R}$-manual/R-devel/library/stats/html/cor.test.html) to calculate the Pearson correlation coefficient (PCC) (25) between the expression level of the intersecting DEGs in the CSCC training dataset and the DElncRNAs, which was performed to construct a co-expression network of DElncRNA and intersecting DEGs. This network was visualized using Cytoscape software (version 3.6.1, https ://cytoscape.org/) (26). An analysis of the Gene Ontology (GO) (22) biological process, and Kyoto Encyclopedia of Genes and Genomes (KEGG) pathway enrichment annotation, based on the Database for Annotation, Visualization and Integrated Discovery (27) (version 6.8, https:// david.ncifcrf.gov/) $(28,29)$, was performed on the intersecting DEGs with $\mathrm{P}<0.05$ as the threshold.

\section{Construction of a prognostic model based on DERs}

Our univariate and multivariate Cox regression analyses, using the survival package in R3.4.4 (version 2.41-1, http://bioconductor.org/packages/survivalr) (30) were used to screen the DERs that were significantly related to overall survival (OS) and prognoses based on the CSCC tumor samples in the training dataset; a $\mathrm{P}<0.05$ was used as the threshold according to the log-rank test. A LASSO Cox regression (31) model, based on the L1-penalized regularization regression algorithm in the penalized package (Version 0.9-50; http://bioconductor.org/packa ges/penalized/) (32) in R3.4.1, was used to screen out the optimized combinations of the prognosis-related signature DERs (the optimized parameter "lambda" in the model was selected and calculated through a 1000 times cross validation likelihood cycle). The Kaplan-Meier (KM) (33) survival curve in the R3.4.1 language survival package (version 2.41-1) (30) was used to evaluate the association between patients OS time and the expression of the optimized DERs. Then, based on the prognostic coefficient of genes in the optimal DER combinations, obtained from the previous regression algorithm, we constructed a risk prediction model, based on the gene expression level in the training dataset, and calculated the risk score of each sample. The prognostic score (PS) was calculated as follows:

$$
\mathrm{PS}=\sum \beta_{\mathrm{DER}} \mathrm{S} \times \operatorname{Exp}_{\mathrm{DERS}}
$$

where $\beta D E R s$ represents the prognostic coefficient of the optimized DERs in the LASSO algorithm, andExp DERs $_{\text {s }}$ represents the expression level of the corresponding DERs in the training dataset.
With the median PS as the cutoff, the samples in the training dataset were separate into high-risk and lowrisk groups, and the correlation between the risk model and prognosis was evaluated by a KM (33) survival curve in the R3.4.1 language survival package (version 2.41-1) (30). Moreover, we extracted the expression value of the target DEGs from the GSE44001 validation dataset. Each sample's PS score was obtained according to the equation described above by using the $\beta$ value from training dataset. The validation dataset samples were divided into the high- and low-risk sample groups based on the PS value of the validation dataset. The survival package (version 2.41-1) and the KM curve method in R3.4.1 (30) was used to evaluate the association between the high- and low-risk groups and the actual survival-related prognosis information for the validation dataset samples.

\section{Screening of independent prognostic clinical factors}

We performed the univariate and multivariate Cox regression analyses using the R3.4.1 language survival package (Version 2.41-1) (34) and screened the independent prognostic clinical factors in CSCC samples from the TCGA dataset with a threshold of $\mathrm{P}<0.05$ from the log-rank test to screen for significant correlations.

To explore the association between the independent prognostic clinical factors and the risk groups, we performed a clinical factor stratification analysis of the selected independent prognostic factors that were significantly correlated: the samples were divided into different groups according to the clinical factors, and a correlation analysis of the risk prognostic models was performed for these different groups.

According to the PS values, the samples in the TCGA training dataset were separatedinto high-risk and lowrisk groups. Then, the limma package was used to analyze the differences between the mRNA expression matrices of the samples in the high- and low-risk groups. Similarly, an $\mathrm{FDR}<0.05$ and $|\operatorname{logFC}|>0.5$ were used as thresholds for determining significant differences. Then, an analysis of the GO (22), BP, and KEGG pathway enrichment annotation based on The DAVID (version 6.8, https:// david.ncifcrf.gov/) $(28,29)$ was performed on the intersecting DEGs with $\mathrm{P}<0.05$ as the threshold.

\section{Results}

\section{Screening of significant DEGs}

With intersections among the TCGA, and GSE44001 datasets, 343 lncRNAs and 15,735 mRNAs were obtained. Then, we divided the samples into the poor prognostic and good prognostic groups, which included 26 and 28 samples, respectively. In all, 454 DERs (197 down-regulated DERs and 257 upregulated DERs) were screened using the limma package (Fig. 1a). 


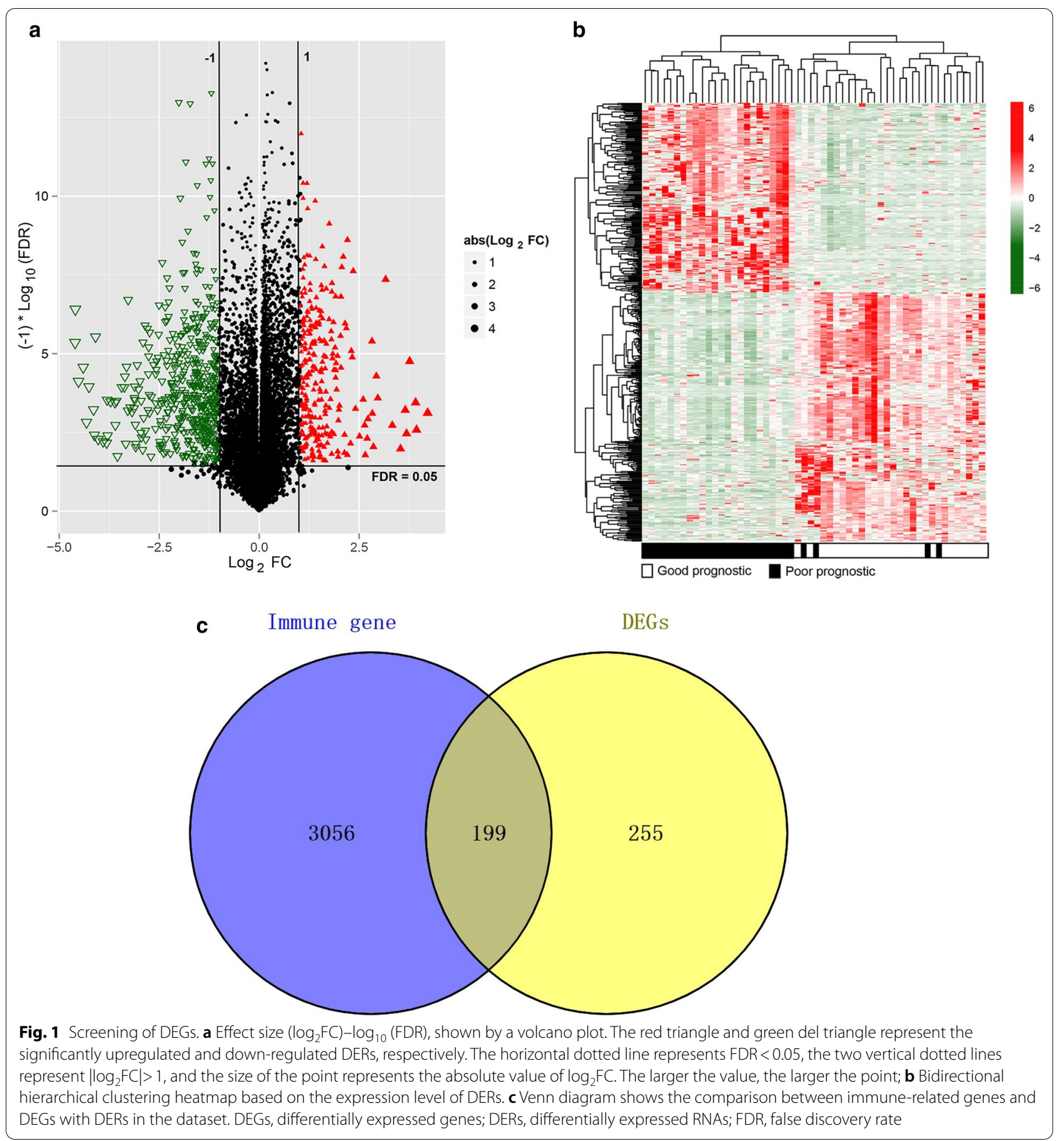

The heatmap showed that the samples were clustered in two different directions (Fig. 1b).

Moreover, 3020 unique genes related to immune GO, 817 unique genes related to immune KEGG, 582 intersecting genes, and 3255 union genes were obtained from the database. When the immune-related genes were compared with the DEG dataset, 199 shared genes were obtained (Fig. 1c) and were used for the next analysis.

\section{Construction of the co-expression network}

The PCC was calculated between the expression level of a gene in the CSCC tumor samples from the TCGA dataset and that of the intersecting DEGs and DElncRNAs. 
In all, 375 pairs of connections were obtained with a cutoff expression correlation coefficient higher than 0.4 , which was used to construct the DElncRNA and DEG intersection co-expression network. As shown in Figs. 2, 5 lncRNAs and 130 mRNAs were included in this network. Interestingly, we observed antisense lncRNAs that were co-expressed with sense mRNAs, such as the RBAKDN-SLC7A10, LINC00158-MS4A1/TNFRSF13BLINC00426, and LINC00158-FCRL1-PIK3CD-AS1 pairs. Moreover, we performed GO and KEGG analyses for the DEGs; overall, 22 BPs and 18 KEGG pathways were screened. The GO analysis indicated that the DEGs were primarily involved in immune response (34 genes, such as $C X C L 2, Z A P 70, C D 27)$, regulation of immune response (22 genes, such as VCAM1, KLRB1, CLEC2D), inflammatory response (21 genes, such as CXCL2, ZAP70, $C D 27)$, and the innate immune response (20 genes, such as ZAP70, CLEC7A). The KEGG pathway analysis revealed that the DEGs were primarily enriched in pathways related to cytokine-cytokine receptor interaction (19 genes, such as CXCL2, TNFRSF13, CD27), TCR signaling pathway (14 genes, such as $Z A P 70, C D 8 A$ ), and Primary immunodeficiency (10 genes, such as ZAP70, TNFRSF13B) (Fig. 2b).

\section{Constructing the prognostic prediction model}

In all, 123 DERs that were markedly associated with CC prognosis were obtained by univariate Cox regression analysis using the cutoff of the log-rank $\mathrm{P}<0.05$. We then obtained 31 independent DEGs that were dramatically related to prognosis by multivariate Cox regression analysis. Subsequently, seven optimized DER groups (RBAKDN, CXCL2, ZAP70, CLEC2D, CD27, KLRB1, $V C A M 1)$ were selected through Cox-Proportional

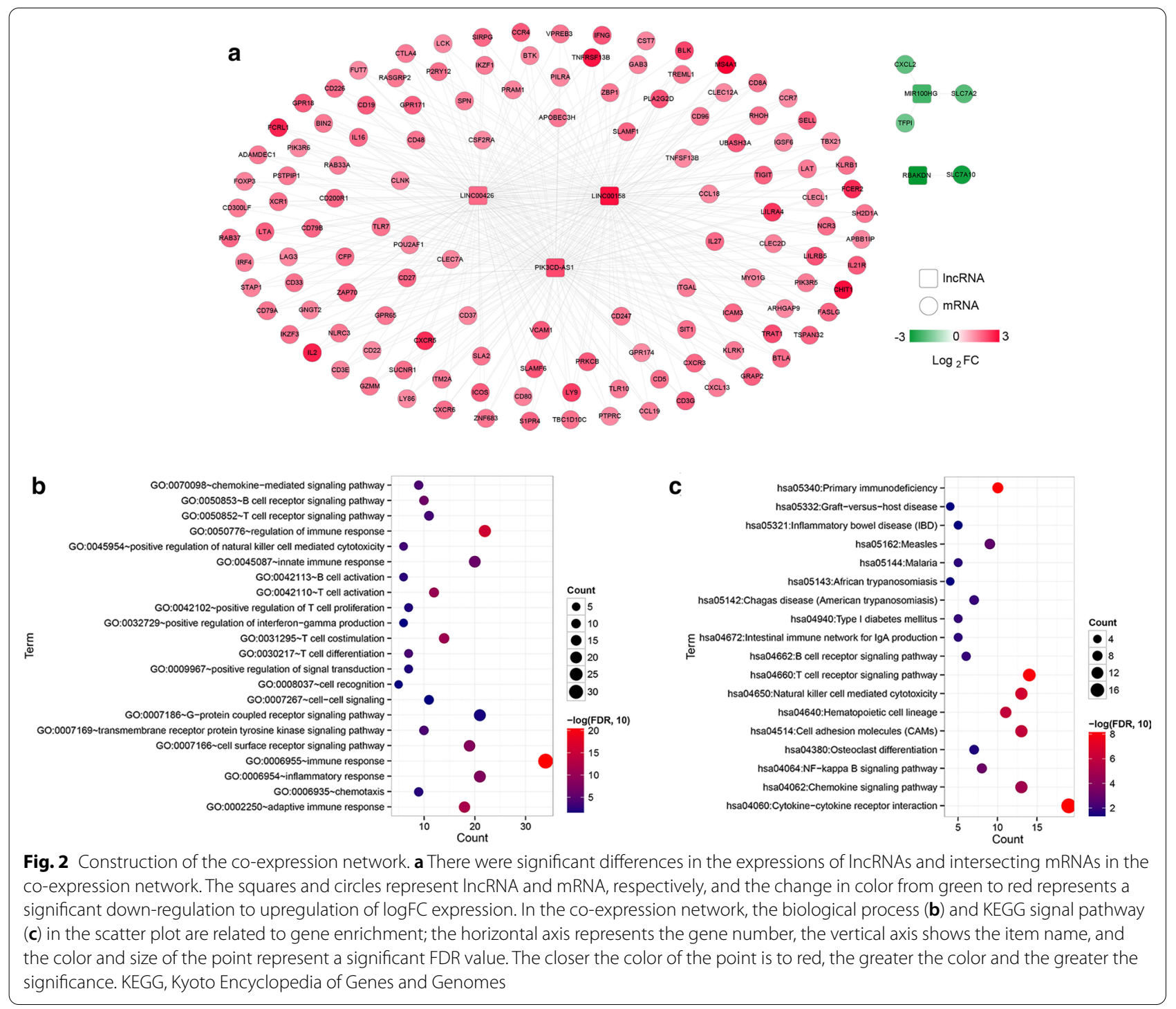


hazard (Cox-PH) regression models on basis of the L1-penalized regularization regression algorithm in penalized the package of $\mathrm{R}$. Among the seven genes, RBAKDN and CXCL2 were risk factors (hazard ratio $(\mathrm{HR})>1$ ), whereas $Z A P 70, C L E C 2 D, C D 27, K L R B 1$, and $V C A M 1$ were identified as protective factors $(\mathrm{HR}<1)$. Additional information on these seven genes is shown in Table 1. Moreover, the KM curves indicated that the low expression of RBAKDN and CXCL2 was associated with good prognosis, while the high expression of $Z A P 70$, $C L E C 2 D, C D 27, K L R B 1$, and VCAM1 was related to better OS time than their high expression (Fig. 3).

\section{Evaluation and comparison of the effectiveness of the prognostic risk prediction model}

The PS model was constructed on basis of the seven optimum DERs, found by LASSO algorithm, and their expression level in the TCGA training dataset. Then, we divided the TCGA training dataset and the GSE44001 validation dataset into high-risk and low- risk groups. The TCGA dataset's KM curves were used to evaluate the connection between the high- and low-risk groups and actual prognostic information for CC. As shown in Fig. 4, we found that samples from low-risk cases in the TCGA dataset had a better survival prognosis $(\mathrm{P}=2.351 \mathrm{e}-04$, $\mathrm{HR}=3.485$ [1.717-7.076], $\mathrm{AUC}=0.906)$; there was a similar trend for the GSE44001 validation dataset $(\mathrm{P}=1.57 \mathrm{e}$ $02, \mathrm{HR}=2.238[1.124-4.454], \mathrm{AUC}=0.799)$. The results revealed a notable correlation between the actual prognosis and the different risk groups obtained after the samples from the TCGA dataset and GSE44001 dataset were divided based on the PS model prediction.

\section{Screening and stratification of independent prognostic clinical factors}

Two were significantly correlated independent prognostic factors (Pathologic N and PS model status) were

\begin{tabular}{|c|c|c|c|c|c|}
\hline \multirow[t]{2}{*}{ Symbol } & \multirow[t]{2}{*}{ Type } & \multicolumn{3}{|c|}{ Univariate Cox regression analysis } & \multirow{2}{*}{$\begin{array}{l}\text { LASSO } \\
\text { coefficient }\end{array}$} \\
\hline & & HR & $95 \% \mathrm{Cl}$ & $P$ value & \\
\hline BAKDN & IncRNA & 11.950 & $4.529-31.54$ & $5.421 \mathrm{E}-07$ & 1.87328 \\
\hline CXCL2 & mRNA & 1.427 & $1.192-1.709$ & $1.098 \mathrm{E}-04$ & 0.35901 \\
\hline ZAP70 & mRNA & 0.493 & $0.323-0.754$ & $1.107 \mathrm{E}-03$ & -0.30060 \\
\hline CLEC2D & mRNA & 0.329 & $0.167-0.651$ & $1.399 \mathrm{E}-03$ & -0.47174 \\
\hline CD27 & mRNA & 0.595 & $0.428-0.827$ & $2.006 \mathrm{E}-03$ & -0.06947 \\
\hline KLRB1 & mRNA & 0.498 & $0.318-0.781$ & $2.403 \mathrm{E}-03$ & -0.03436 \\
\hline VCAM1 & mRNA & 0.542 & $0.363-0.809$ & $2.768 \mathrm{E}-03$ & -0.14466 \\
\hline
\end{tabular}

$\mathrm{HR}$, hazard ratio; $\mathrm{Cl}$, confidence interval screened by univariate and multivariate Cox regression analyses in the TCGA dataset (Table 2). We found that the lower the Pathologic N, the better the prognosis with respect to normal platelet indicators in CSCC tumor patients, which is consistent with the actual situation. We then separated the training dataset samples into the NO and N1 sample groups, according to the Pathologic N status, and then analyzed the correlation between the prediction results of the PS prognostic model and the actual prognosis in each stratified sample dataset (Fig. 5). The KM curves of the Pathologic N status revealed that the samples in the N0 group had a better OS $(\mathrm{P}=1.33 \mathrm{e}-03)$. Moreover, the KM curves of the Pathologic N0 and Pathologic N1 groups showed that the low-risk group had a better OS $(\mathrm{P}=3.772 \mathrm{e}-03 ; \mathrm{P}=7.755 \mathrm{e}-01)$.

\section{Functional analysis of high- and low-risk key genes}

According to the PS value, the TCGA samples were separated into the high-risk and low-risk groups. In all, 254 DEGs (185 significantly down-regulated and 69 significantly upregulated genes) were screened using the limma package to analyze the differences between the expression matrices of the high- and low-risk group samples from the TCGA dataset (Fig. 6a). The heatmap showing the DEGs' expression based on the risk score is shown in (Fig. 6b). The heatmap revealed that the DEGs' expression level was significantly altered as the risk score changed increased. Then, a GO, BP, and KEGG pathways analysis was performed to determine the DEGs with a cutoff of $\mathrm{P}<0.05$. In all, 15 BPs and 7 KEGG pathways were screened. The GO analysis indicated that the DEGs were primarily involved in adaptive immune response (13 genes, such as TNFRSF13B, THEMIS) and immune response (18 genes, such as CXCL2) (Fig. 6c); the KEGG pathway analysis revealed that DEGs were primarily enriched in pathways related to cytokine-cytokine receptor interaction (CXCL2, TNFRSF13B) (Fig. 6c).

\section{Discussion}

Although there has been substantial progress in the early diagnosis and treatment of CSCC, the incidence of this disease is still high, and it has a low diagnosis rate as well as a poor prognosis $(35,36)$. In our study, we used comprehensive bioinformatics analyses to identify seven prognosis-related signature genes (RBAKDN, CXCL2, ZAP70, CLEC2D, CD27, KLRB1, VCAM1). Their expression was significantly related to OS in CSCC patients. In addition, the risk score of the seven-gene feaure expressed its superior ability to categorize CSCC patients into high-risk and low-risk groups with markedly different OS in the training and validation datasets.

Additionally, to further distinguish the important genes that participate in the prognostic model, we constructed 


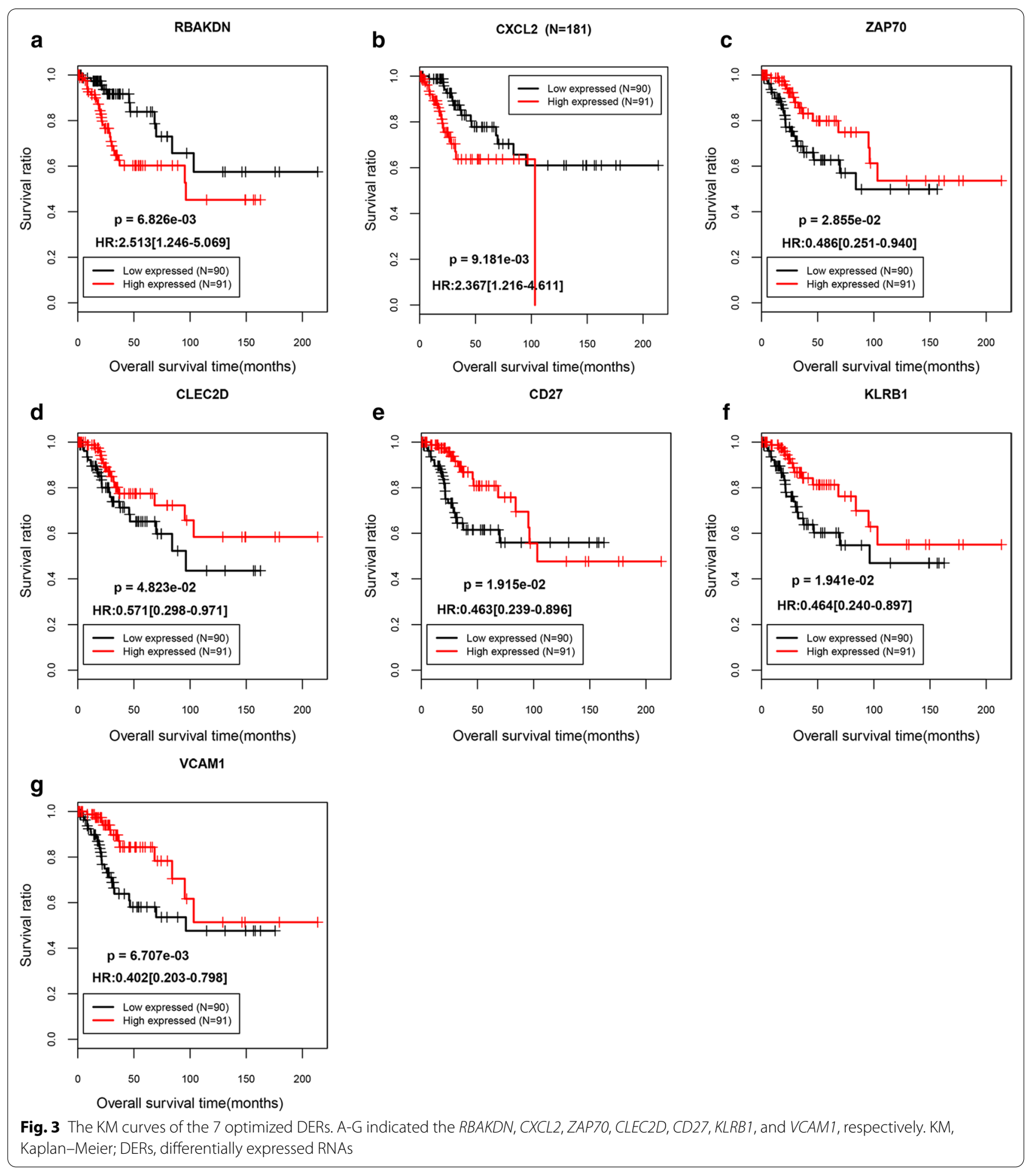

a protein-protein interaction (PPI) network. The results revealed that three hub genes were screened in the PPI network, MS4A1, TNFRSF13B, and FCRL1, which are significantly expressed. TNFRSF13B, a member of the TNF receptor superfamily, which occupied an important position in the proliferation and progression of cancer cell. Abo-Elfadl MT et al. (37) reported that TNFRSF13B silencing could be a therapeutic target for breast cancer subtype. Fc receptor-like $1(F C R L 1)$ is a novel B cell receptor (BCR) co-receptor. Zhao et al. (38) revealed a 

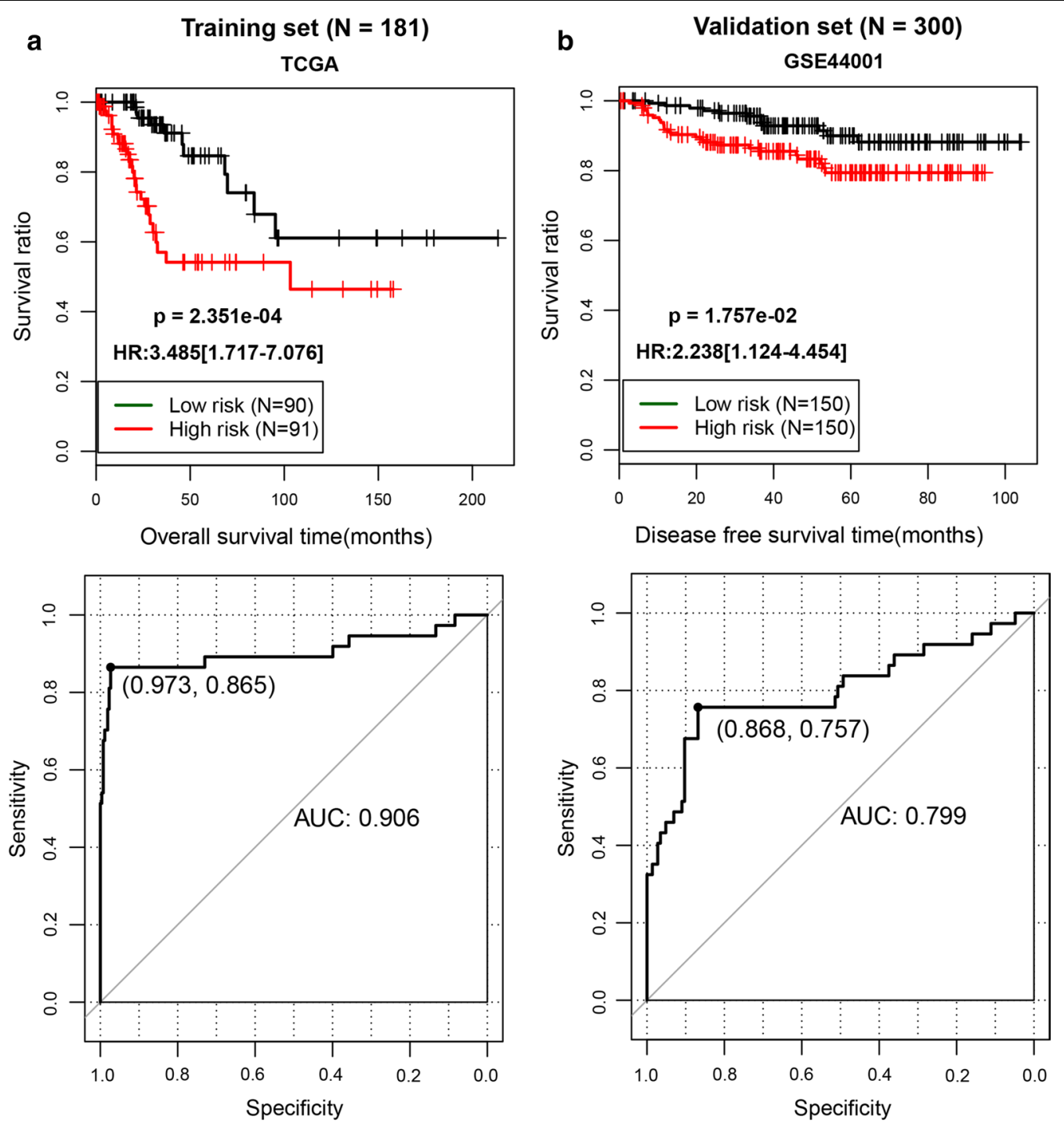

Fig. 4 Upper panel:TCGA (a) and GSE44001 (b) samples are based on the KM curve of the PS prediction model and the prognosis; the green and red curves represent low- and high-risk samples, respectively. Lower panel: ROC curve of the prediction results based on the PS prognostic model. The numbers in brackets represent the specificity and sensitivity corresponding to the ROC curve. TCGA, The Cancer Genome Atlas; KM, KaplanMeier; PS, prognostic score; ROC, receiver operating characteristic

vital BCR signal enhancement role of FCRL1 via its internal B cell immune synapse recruitment and subsequent c-Abl recruitment on BCR cross-linking. Furthermore, KEGG and GO analyses expressed that the genes were enriched in the immune response pathway. In particular, these results suggest the importance of genes associated with the immune response.

Moreover, among the seven prognosis-related signature genes, RBAKDN and CXCL2 were both determined to be risk factors. A previous investigation found that the chemokine (C-X-C motif) ligand 2 (CXCL2) was originally characterized as a neutrophil chemokine.
Specifically, CXCL2 could improved the expansion of monocytic MDSCs (mo-MDSCs) a subtype of MDSCs. Shi et al. (39) indicated that CXCL2 was expressed in tumor cells and tumor-infiltrating CD11b myeloid cells, which shows $C X C L 2$ 's novel role in increasing moMDSC generation by favoring the differentiation of bone marrow cells in tumor-bearing conditions. This suggests that inhibiting the levels of CXCL1 and CXCL2 could reduce mo-MDSC generation and promote host immunosurveillance. Zhang et al. (40) reported that A-kinaseinteracting protein 1 is crucial in $\mathrm{CC}$ angiogenesis and growth because it functions to elevate the levels of the 
Table 2 Clinical factor screening information sheet

\begin{tabular}{|c|c|c|c|c|c|c|c|}
\hline \multirow[t]{2}{*}{ Clinical characteristics } & \multirow[t]{2}{*}{ TCGA $(\mathrm{N}=181)$} & \multicolumn{3}{|c|}{ Uni-variables cox } & \multicolumn{3}{|c|}{ Multi-variables cox } \\
\hline & & HR & $95 \% \mathrm{Cl}$ & $\mathbf{P}$ & HR & $95 \% \mathrm{Cl}$ & $\mathbf{P}$ \\
\hline Age (years, mean \pm sd) & $47.06 \pm 13.83$ & 1.005 & $0.981-1.029$ & $6.93 E-01$ & - & - & - \\
\hline Neoplasm histologic grade (G1/G2/G3/-) & $11 / 77 / 77 / 16$ & 1.219 & $0.655-2.269$ & 5.30E-01 & - & - & - \\
\hline Pathologic M (M0/M1/-) & 79/3/99 & 11.060 & $1.130-108.2$ & $9.48 \mathrm{E}-03$ & 5.960 & $0.598-59.39$ & $1.28 \mathrm{E}-01$ \\
\hline Pathologic N (N0/N1/-) & $97 / 30 / 54$ & 3.410 & $1.541-7.547$ & $1.33 E-03$ & 3.238 & $1.468-7.142$ & $3.61 E-03$ \\
\hline Pathologic T (T1/T2/-) & $99 / 50 / 32$ & 1.263 & $0.584-2.729$ & $5.52 \mathrm{E}-01$ & - & - & - \\
\hline Pathologic stage $(I / I)$ & $123 / 58$ & 0.820 & $0.396-1.696$ & 5.87E-01 & - & - & - \\
\hline Number of pregnancies $(0 / 1 / 2 / 3 /$ over $3 /-)$ & $10 / 18 / 30 / 28 / 75 / 20$ & 1.028 & $0.794-1.333$ & $8.31 \mathrm{E}-01$ & - & - & - \\
\hline Radio-therapy (yes/no/-) & $105 / 53 / 23$ & 1.190 & $0.571-2.479$ & $6.42 \mathrm{E}-01$ & - & - & - \\
\hline RS model status (high/low) & $90 / 91$ & 3.485 & $1.717-7.076$ & $2.35 E-04$ & 2.518 & $1.122-5.651$ & $2.51 \mathrm{E}-02$ \\
\hline Vital status (dead/alive) & $38 / 143$ & - & - & - & - & - & - \\
\hline Overall survival time (months, mean \pm sd) & $37.83 \pm 40.02$ & - & - & - & - & - & - \\
\hline
\end{tabular}

$\mathrm{HR}$, hazard ratio; $\mathrm{Cl}$, confidence interval; $\mathrm{N}$, number
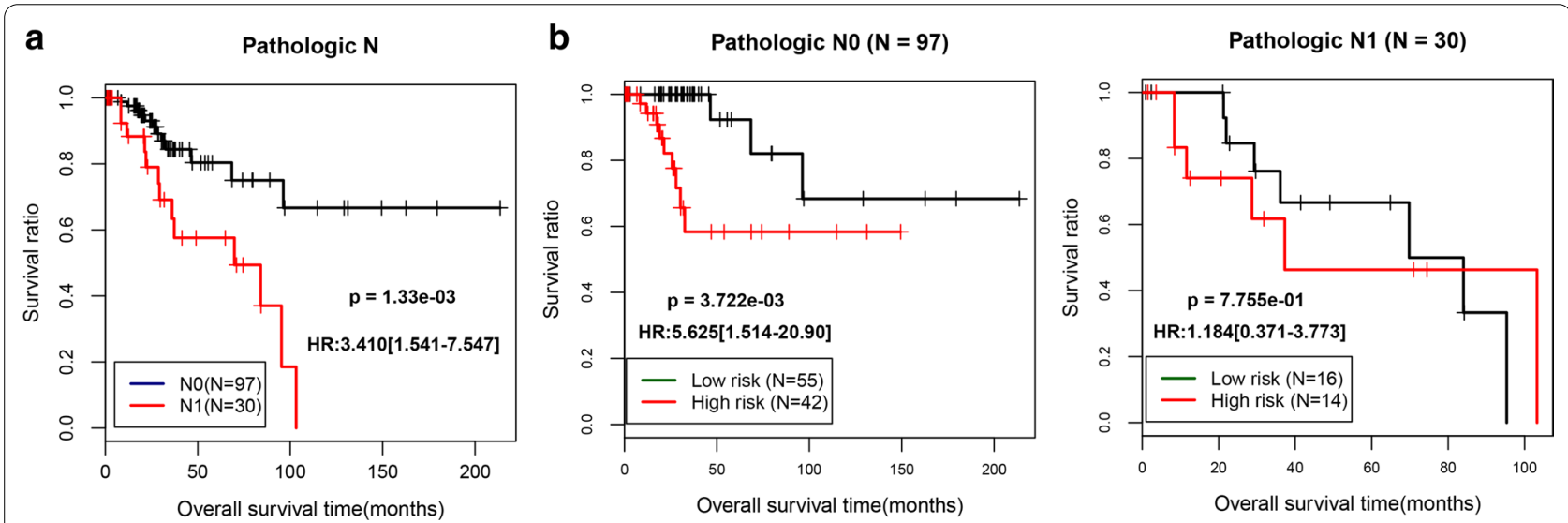

Fig. 5 Screening and stratification of independent prognostic clinical factors. (a) The KM curve of the prognostic correlation of the Pathologic $\mathrm{N}$ stage is shown in the TCGA samples, and the black and red curves show the samples that were Pathologic N0 and N1 stages, respectively. (b) The samples in the demographic Pathologic NO group are based on the KM curve of the PS prediction model and the prognosis; the black and red curves represent the low- and high-risk samples, respectively. KM, Kaplan-Meier; TCGA, The Cancer Genome Atlas; PS prognostic score

NF-kB-dependent chemokines CXCL1, CXCL2, and CXCL8. Zheng et al. (41) revealed that chemokine CXCL2 induced lung cancer-associated transcript 1 (LUCAT1) overexpression and that the CXCL2 $\beta$ axis is a potential therapeutic target and molecular biomarker for clear cell renal cell carcinoma (ccRCC). All these results indicated that $C X C L 2$ could induce the production of adverse factors, which could contribute to CSCC's poor prognosis. These results are consistent with ours and suggest that CXCL2 is highly valuable for predicting the survival and prognosis of patients with CSCC. However, no studies have considered RBAKDN's possible role in cancer.

To further explore the potential mechanism of immune-related genes, we observed that high-risk key genes were associated with the cytokine-cytokine receptor interaction pathway. The TRAIL/TRAIL-R system was regulated by Macrophages and neutrophils via cytokines to remove cancer cells (42). Li et al. (43) indicated the bistability of the network between cytokines and tumor immunity. This model has shown that tumors can take advantage of this bistability to improve immunosuppression. Eliminating this interaction means the immune system can return to an immune-boosting state. These results suggest that certain differentially expressed immune-related genes involved in cytokine-cytokine receptor interaction contribute to longer OS. 


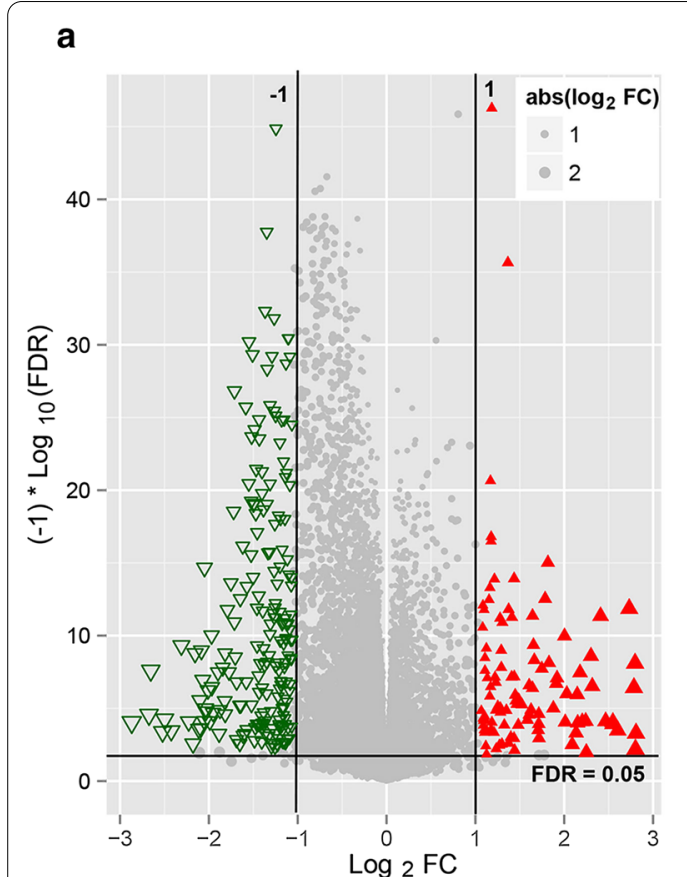

\section{b}

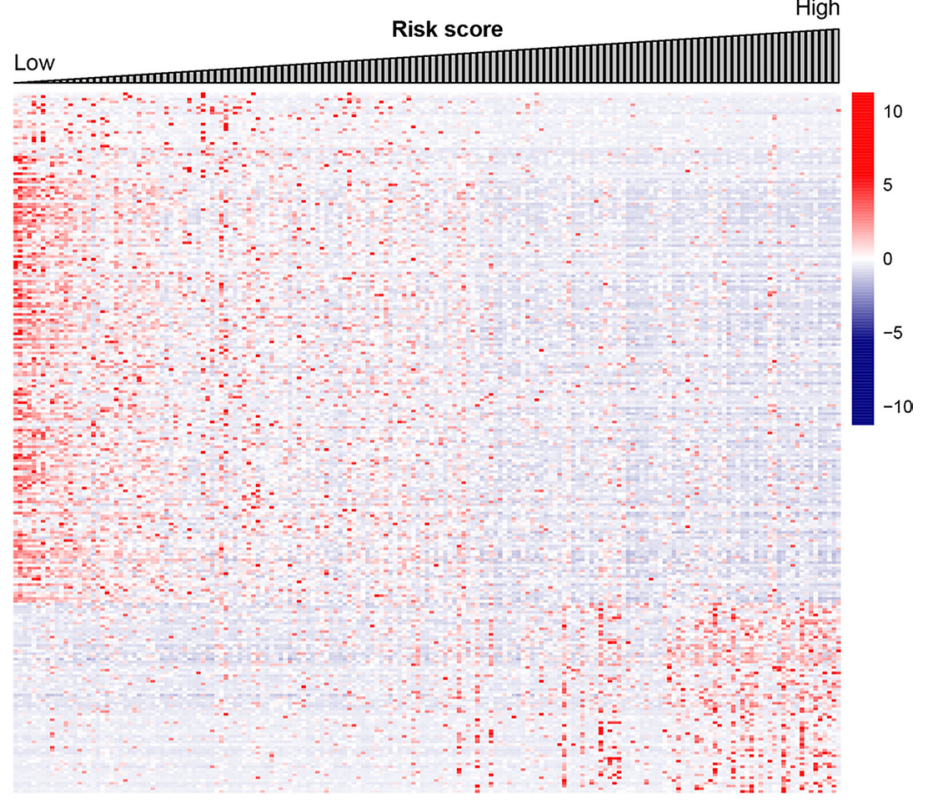

C

hsa05340:Primary immunodeficiency -

hsa05134:Legionellosis -

hsa04940:Type I diabetes mellitus -

hsa04672:Intestinal immune network for IgA production -

hsa04640:Hematopoietic cell lineage -

hsa04062:Chemokine signaling pathway -

hsa04060:Cytokine-cytokine receptor interaction -

GO:0071805 potassium ion transmembrane transport -

GO:0070098 chemokine-mediated signaling pathway -

GO:0050776 regulation of immune response -

E GO:0034765 regulation of ion transmembrane transport -

๕ᄄ

GO:0032496 response to lipopolysaccharide -

GO:0019221 cytokine-mediated signaling pathway -

GO:0007601 visual perception -

GO:0007267 cell-cell signaling -

GO:0007155 cell adhesion -

GO:0006968 cellular defense response -

GO:0006959 humoral immune response -

GO:0006955 immune response -

GO:0006954 inflammatory response -

GO:0006935 chemotaxis -

GO:0002250 adaptive immune response
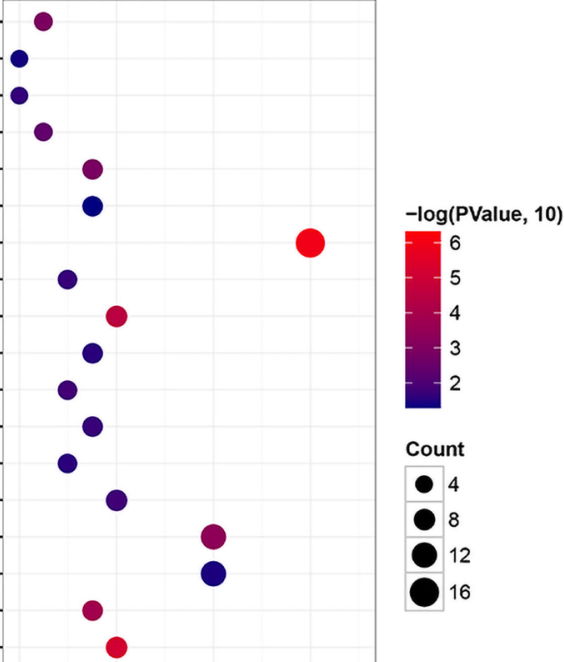

Count

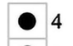

8

16

Fig. 6 Functional analyses of high- and low-risk key genes. a In the effect size $\left(\log _{2} \mathrm{FC}\right)$ - $\log _{1} 0$ (FDR) volcano map, the red triangle, and green triangle represent significantly upregulated and down-regulated DEGs, respectively, the horizontal dotted line represents FDR $<0.05$, the two vertical dotted lines represent $\left|\log _{2} F C\right|>1$, and the point size represents the absolute value of $\log _{2}$ FC. The larger the value, the larger the point; $\mathbf{b}$ Based on the high- and low-risk groups, the changes in DEGs with the corresponding diagnostic scores from a low to a high expression level. c The horizontal axis represents the number of genes, the vertical axis represents the name of the item, and the color and size of the point represent the significant FDR value. The closer the color of the point is to red, the greater the color and the greater the significance. DEGs, differentially expressed genes; DERs, differentially expressed RNAs; FDR, false discovery rate 


\section{Conclusions}

In summary, we identified seven prognosis-related signature genes (RBAKDN, CXCL2, ZAP70, CLEC2D, CD27, $K L R B 1, V C A M 1)$, the expression of which significantly correlated with OS in CSCC patients. Also, the risk score of the seven-gene signature demonstrated superior ability to divide CSCC patients into high-risk and low-risk groups, each of which had a markedly different OS in the training dataset and validation datasets. Two significantly correlated independent prognostic factors (Pathologic N and PS model status) were screened by univariate and multivariate Cox regression analyses in the TCGA dataset. To further explore the potential mechanism of immune-related genes, we observed that high-risk key genes were related to cytokine-cytokine receptor interaction.

\section{Abbreviations}

BCR: B cell receptor; CC: Cervical cancer; ccRCC: Cell renal cell carcinoma; CIN Cervical intraepithelial neoplasia; CSCC: Cervical squamous cell carcinoma; CXCL2: Chemokine (C-X-C motif) ligand 2; DEIRGs: Differentially expressed immune-related genes; FCRL1: Fc receptor-like 1; GO: Gene ontology; KEGG: Kyoto encyclopedia of genes and genomes; LUCAT1: Lung cancer-associated transcript 1; mo-MDSCs: Monocytic MDSCs; OS: Overall survival; PCC: Pearson correlation coefficient; PS: Prognostic score; TCGA: The cancer genome Atlas; TCR: T cell receptor.

\section{Acknowledgements}

None.

\section{Authors' contributions}

JRW and RQ were responsible for the conception and design of the research, and drafting the manuscript. LC, CY and ZQS performed the data acquisition, analysis and interpretation. RQ participated in the design of the study and performed the statistical analysis. All authors read and approved the final manuscript.

\section{Funding}

None.

\section{Availability of data and materials}

The datasets supporting the conclusions of this article are available in the [GSE44001] and [TCGA] repository, [https://www.ncbi.nlm.nih.gov/geo/query /acc.cgi?acc=GSE44001] and [https://xenabrowser.net/datapages/?cohor t=GDC\%20TCGA\%20Cervical\%20Cancer\%20(CESC)\&removeHub=https \%3A\%2F\%2Fxena.treehouse.gi.ucsc.edu\%3A443].

Ethics approval and consent to participate Not applicable.

\section{Consent for publication}

Not applicable.

\section{Competing interests}

The authors declare that they have no competing interests.

Received: 11 August 2020 Accepted: 25 January 2021

Published online: 15 February 2021

\section{References}

1. Torre LA, Siegel RL, Ward EM, Jemal A. Global cancer incidence and mortality rates and trends - an update. Cancer Epidemiol Biomark Prev. 2016:25(1):16-27.
2. Siegel RL, Miller KD, Jemal A. Cancer statistics, 2018. CA Cancer J Clin. 2018:68:7-30

3. Solís JG, Briones-Torres TI. Prevalence of intraepithelial lesion in cervical screening cytology in a First-level Care Unit. Rev Med Inst Mex Seguro Soc. 2018:56(2):167-72.

4. Zhu AX, Finn RS, Edeline J, Cattan S, Ogasawara S, Palmer D, et al. Pembrolizumab in patients with advanced hepatocellular carcinoma previously treated with sorafenib (KEYNOTE-224): a non-randomised, open-label phase 2 trial. Lancet Oncol. 2018;19:940-52.

5. Otter SJ, Chatterjee J, Stewart AJ, Michael A. The role of biomarkers for the prediction of response to checkpoint immunotherapy and the rationale for the use of checkpoint immunotherapy in cervical cancer. Clin Oncol (R Coll Radiol). 2019;31(12):834-43.

6. Zhao S, Yu M. MMP1Identification of as a potential prognostic biomarker and correlating with immune infiltrates in cervical squamous cell carcinoma. DNA Cell Biol. 2020;39(2):1-18.

7. Karpathiou G, Chauleur C, Mobarki M, Peoc'h M. The immune checkpoints CTLA-4 and PD-L1 in carcinomas of the uterine cervix. Pathol Res Pract. 2020;216(1):1-16

8. Wang Q, Li P, Wu W. A systematic analysis of immune genes and overall survival in cancer patients. BMC Cancer. 2019;19(1):1225.

9. Topalian SL, Drake CG, Pardoll DM. Immune checkpoint blockade: a common denominator approach to cancer therapy. Cancer Cell. 2015:27(4):450-61.

10. Mao Y, Fu Z, Dong L, Zheng Y, Dong J, Li X. Identification of a 26-IncRNAs risk model for predicting overall survival of cervical squamous cell carcinoma based on integrated bioinformatics analysis. DNA Cell Biol. 2019;38(4):322-32.

11. Zhou YH, Fan WF, Deng J, Xi HL. Establishment and analysis of the prediction model for cervical squamous cell carcinoma. Eur Rev Med Pharm Sci. 2017;21(22):5042-8.

12. He H, Liu X, Liu Y, Zhang M, Lai Y, Hao Y, et al. Human papillomavirus E6/ E7 and long noncoding RNA TMPOP2 mutually upregulated gene expression in cervical cancer cells. J Virol. 2019;93(8):undefined.

13. Haddad G, Lorenzen JM. Biogenesis and function of circular RNAs in health and in disease. Front Pharmacol. 2019;10:428.

14. Wright AA, Howitt BE, Myers AP, Dahlberg SE, Palescandolo E, Van Hummelen $P$, et al. Oncogenic mutations in cervical cancer: genomic differences between adenocarcinomas and squamous cell carcinomas of the cervix. Cancer. 2013;119(21):3776-83.

15. Tornesello ML, Annunziata C, Buonaguro L, Losito S, Greggi S, Buonaguro FM. TP53 and PIK3CA gene mutations in adenocarcinoma, squamous cell carcinoma and high-grade intraepithelial neoplasia of the cervix. J Transl Med. 2014;12:255.

16. Tian W, Lei N, Guo R, Yuan Z, Chang L. Long non-coding RNA DANCR promotes cervical cancer growth via activation of the Wnt/ $\beta$-catenin signaling pathway. Cancer Cell Int. 2020;20(61).

17. Barrett T, Troup DB, Wilhite SE, Ledoux P, Rudnev D, Evangelista C, et al. NCBI GEO: mining tens of millions of expression profiles-database and tools update. Nucleic Acids Res. 2007;35:D760-5.

18. Lee Y-Y, Kim T-J, Kim J-Y, Choi CH, Do I-G, Song SY, et al. Genetic profiling to predict recurrence of early cervical cancer. Gynecol Oncol. 2013;131(3):650-4

19. Volders P-J, Helsens K, Wang X, Menten B, Martens L, Gevaert K, et al. LNCipedia: a database for annotated human IncRNA transcript sequences and structures. Nucleic Acids Res. 2013;41:D246-51.

20. Mathew W. A short guide to long non-coding RNA gene nomenclature. Hum Genomics. 2014:9.7.

21. Li C, Kuang L, Zhu B, Chen J, Wang X, Huang X. Identification of prognostic risk factors of acute lymphoblastic leukemia based on mRNA expression profiling. Neoplasma. 2017;64(4):494-501.

22. Ritchie ME, Phipson B, Wu D, Hu Y, Law CW, Shi W, et al. limma powers differential expression analyses for RNA-sequencing and microarray studies. Nucleic Acids Res. 2015;43(7):e47.

23. Wang L, Cao C, Ma Q, Zeng Q, Wang H, Cheng Z, et al. RNA-seq analyses of multiple meristems of soybean: novel and alternative transcripts, evolutionary and functional implications. BMC Plant Biol. 2014;14:169.

24. Eisen MB, Spellman PT, Brown PO, Botstein D. Cluster analysis and display of genome-wide expression patterns. Proc Natl Acad Sci USA. 1998;95(25):14863-8. 
25. Eberly LE. Correlation and simple linear regression. Methods Mol Biol. 2007;227(3):617-22.

26. Shannon P, Markiel A, Ozier O, Baliga NS, Wang JT, Ramage D, et al. Cytoscape: a software environment for integrated models of biomolecular interaction networks. Genome Res. 2003;13(11):2498-504.

27. Zheng-Bradley X, Streeter I, Fairley S, Richardson D, Clarke L, Flicek P, et al. Alignment of 1000 genomes project reads to reference assembly GRCh38. Gigascience. 2017;6(7):1-8.

28. Huang DW, Sherman BT, Lempicki RA. Systematic and integrative analysis of large gene lists using DAVID bioinformatics resources. Nat Protoc. 2009;4(4):44-57.

29. Huang DW, Sherman BT, Lempicki RA. Bioinformatics enrichment tools: paths toward the comprehensive functional analysis of large gene lists. Nucleic Acids Res. 2009;37(1):1-13.

30. Wang P, Wang Y, Hang B, Zou X, Mao J-H. A novel gene expression-based prognostic scoring system to predict survival in gastric cancer. Oncotarget. 2016.

31. Tibshirani R. The lasso method for variable selection in the Cox model. Stat Med. 1997.

32. Goeman JJ. L1 penalized estimation in the Cox proportional hazards model. Biom J. 2010.

33. Li T, Wernersson R, Hansen RB, Horn H, Mercer J, Slodkowicz G, et al. A scored human protein-protein interaction network to catalyze genomic interpretation. Nat Methods. 2017;14:61-4.

34. Wang P, Wang Y, Hang B, Zou X, Mao J-H. A novel gene expression-based prognostic scoring system to predict survival in gastric cancer. Oncotarget. 2016;7(34):55343-51.

35. Chang S, Sun L, Feng G. SP1-mediated long noncoding RNA POU3F3 accelerates the cervical cancer through miR-127-5p/FOXD1. Biomed Pharmacother. 2019;117:109-33.

36. Tanjasiri SP, Mouttapa M, Sablan-Santos L, Weiss JW, Chavarria A, Lacsamana JD, et al. Design and outcomes of a community trial to increase pap testing in pacific islander women. Cancer Epidemiol Biomarkers Prev. 2019;28(9):1435-42.

37. Abo-Elfadl MT, Gamal-Eldeen AM, Ismail MF, Shahin NN. Silencing of the cytokine receptor TNFRSF13B: a new therapeutic target for triple-negative breast cancer. Cytokine. 2020;125:154790.

38. Zhao X, Xie H, Zhao M, Ahsan A, Li X, Wang F, et al. Fc receptor-like 1 intrinsically recruits $c-A b l$ to enhance $B$ cell activation and function. Sci Adv. 2019;5(7):315.

39. Shi $H$, Han $X$, Sun $Y$, Shang $C$, Wei $M, B a$ X et al. Chemokine (C-X-C motif) ligand 1 and CXCL2 produced by tumor promote the generation of monocytic myeloid-derived suppressor cells. Cancer Sci. 2018;109(12):3826-39.

40. Zhang W, Wu Q, Wang C, Yang L, Liu P, Ma C. AKIP1 promotes angiogenesis and tumor growth by upregulating CXC-chemokines in cervical cancer cells. Mol Cell Biochem. 2018;448:311-20.

41. Zheng Z, Zhao F, Zhu D, Han J, Chen H, Cai Y, et al. Long non-coding RNA LUCAT1 promotes proliferation and invasion in clear cell renal cell carcinoma through AKT/GSK-3 $\beta$ signaling pathway. Cell Physiol Biochem. 2018;48(3):891-904

42. de Looff M, de Jong S, Kruyt FAE. Multiple interactions between cancer cells and the tumor microenvironment modulate TRAIL Signaling: implications for TRAIL receptor targeted therapy. Front Immunol. 2019;10:1530.

43. Li T, Fan J, Wang B, Traugh N, Chen Q, Liu JS, et al. TIMER: a web server for comprehensive analysis of tumor-infiltrating immune cells. Cancer Res. 2017;77(21):e108-10.

\section{Publisher's Note}

Springer Nature remains neutral with regard to jurisdictional claims in published maps and institutional affiliations.
Ready to submit your research? Choose BMC and benefit from:

- fast, convenient online submission

- thorough peer review by experienced researchers in your field

- rapid publication on acceptance

- support for research data, including large and complex data types

- gold Open Access which fosters wider collaboration and increased citations

- maximum visibility for your research: over $100 \mathrm{M}$ website views per year

At BMC, research is always in progress.

Learn more biomedcentral.com/submissions 